\title{
Research Article \\ Regular and Irregular Sampling Theorem for Multiwavelet Subspaces
}

\author{
Liu Zhanwei, ${ }^{1}$ Hu Guoen, ${ }^{2}$ and Wu Guochang ${ }^{3}$ \\ ${ }^{1}$ School of Information Engineering, Zhengzhou University, Zhengzhou 450001, China \\ ${ }^{2}$ Department of Applied Mathematics, Zhengzhou Information Science and Technology Institute, \\ Zhengzhou 450002, China \\ ${ }^{3}$ College of Information, Henan University of Finance and Economics, Zhengzhou 450002, China \\ Correspondence should be addressed to Liu Zhanwei, changgengliu@163.com
}

Received 5 March 2010; Accepted 26 July 2010

Academic Editor: Victoria Vampa

Copyright (c) 2010 Liu Zhanwei et al. This is an open access article distributed under the Creative Commons Attribution License, which permits unrestricted use, distribution, and reproduction in any medium, provided the original work is properly cited.

\begin{abstract}
We study the sampling theorem for frames in multiwavelet subspaces. Firstly, a sufficient condition under which the regular sampling theorem holds is established. Then, notice that irregular sampling is also useful in practice; we consider the general cases of the irregular sampling and establish a general irregular sampling theorem for multiwavelet subspaces. Finally, using this generalized irregular sampling theorem, we obtain an estimate for the perturbations of regular sampling in shift-invariant spaces.
\end{abstract}

\section{Introduction}

At the present time the sampling theorem plays a crucial role in signal processing and communication, as it establishes an equivalence between discrete signals and analogue (continuous) signals. For a band-limited signal, the classical Shannon sampling theorem provides an exact representation by its uniform samples with a sampling rate higher than its Nyquist rate. But there exist several problems. Firstly, real-world signals or images are never exactly band-limited. Secondly, there is no such device as an ideal (antialiasing or reconstruction) low-pass filter. Thirdly, Shannon reconstruction formula is rarely used in practice (especially with images) because of the slow decay of the sinc function. Therefore, this classical Shannon sampling theorem has been generalized to many other forms.

Extensions of Shannon sampling theorem to scalar wavelets can be found in [15], but a scalar wavelet cannot have the orthogonality, compact support, and symmetry at the same time (except the Haar wavelet). It is a disadvantage for signal processing. 
Meanwhile, multiwavelets have attracted much attention in the research community, since multiwavelet has more desired properties than any scalar wavelet function, such as orthogonality, short compact support, symmetry, high approximation order, and so on. The first orthogonal multiwavelet with symmetry, approximation order, and compact support was presented by Geronimo et al. [6]. In addition, the sampling theorems for multiwavelet subspaces were studied in [7-10]. The authors of $[7,9]$ presented the construction of compactly supported orthogonal multiscaling functions that are continuously differentiable and cardinal. The scaling functions thereby support a Shannon-like sampling theorem. However, the multiwavelets of $[7,9]$ do not have symmetry. It is not good for digital signal processing and image compression. They also did not study the sampling theorem for frame in multiwavelet subspaces, which is very important in application.

A reconstruction from more general sets of points is necessary if the measurements cannot be made at uniform points. Hence, irregular sampling is also useful in practice. In [11], the authors obtained a Feichtinger-Grochenig iterative algorithm based on the quasiinterpolation projection procedure to recover signals from irregular samples for multiwavelet subspaces. However, the maximal allowable gap between two sampling points needed for reconstructing a function from its samples was not obtained, which was supposed to exist in theory. The authors of [10] generalized the multiwavelet sampling theorem by reproducing a kernel and derived an estimate for the perturbations of uniform noninteger sampling in shift-invariant spaces, but their results just based on the Riesz basis.

In our paper, we will show a sufficient condition for regular sampling theorem to hold in multiwavelet subspaces for frames. Notice that a reconstruction from more general sets of points is necessary if the measurements cannot be made an uniform; we establish the irregular sampling theorems in multiwavelet subspaces. Finally, an estimate for the perturbations of regular sampling in shift-invariant spaces is derived.

This paper is organized as follows. Section 2 contains some definitions in this correspondence. Also, we review some relative notations. In Section 3, we discuss general uniform noninteger sampling and obtain a sufficient condition for uniform noninteger sampling theorem to hold. In Section 4, an irregular sampling theorem in general multiwavelet subspaces is established. Finally, by applying the result in Section 4, we estimate the perturbations of uniform noninteger sampling in shift-invariant spaces.

\section{Preliminary}

We now introduce some notations used in this correspondence.

$C(R)$ is the space of continuous function.

The shift-invariant closed subspace $V_{0}$ generated by $\left\{\phi_{1}, \phi_{2}, \ldots, \phi_{r}\right\}$

$$
V_{0}=\overline{\operatorname{span}\left\{\phi_{i}(\cdot-k): 1 \leq i \leq r, k \in Z\right\}} \subset L^{2}(R) .
$$

For a function $f \in L^{2}(R)$, we denote by $\langle f\rangle$ the minimal closed shift invariant subspace that contains $f$.

Let

$$
\mathbf{f}=\left[f_{1}, f_{2}, \ldots, f_{r}\right]^{T}
$$


denote vector (we denote vectors and matrices in this paper in boldface). The integration $\int_{R} \mathbf{f}(x) d x$ is defined as

$$
\int_{R} \mathbf{f}(x) d x=\left[\int_{R} f_{1}(x) d x, \int_{R} f_{2}(x) d x, \ldots, \int_{R} f_{r}(x) d x\right]^{T}
$$

The Fourier transform of vector $\mathbf{f}$ is defined by

$$
\widehat{\mathbf{f}}(\omega)=\int_{R} \mathbf{f}(x) e^{-i x \omega} d x
$$

The inverse Fourier transform of vector $\mathbf{f}$ is written by

$$
\check{\mathbf{f}}(x)=\frac{1}{2 \pi} \int_{R} \mathbf{f}(\omega) e^{i x \omega} d \omega .
$$

$Z_{f}(x, \omega)=\sum_{n \in Z} f(x+n) e^{-i n \omega}$ is the Zak transform of function $f$. The Zak transform of vector $\mathrm{f}$ is defined by

$$
Z_{\mathbf{f}(x, \omega)}=\left[Z_{f_{1}}(x, \omega), Z_{f_{2}}(x, \omega), \ldots, Z_{f_{r}}(x, \omega)\right]^{T} .
$$

A collection of elements $\left\{\phi_{i}(\cdot-k): 1 \leq i \leq r, k \in Z\right\}$ in a Hilbert space $H$ is called a frame if there exist constants $A$ and $B, 0<A \leq B<\infty$, such that

$$
A\|f\|^{2} \leq \sum_{k \in Z} \sum_{i=1}^{r}\left|\left\langle f, \phi_{i}(\cdot-k)\right\rangle\right|^{2} \leq B\|f\|^{2}, \quad \forall f \in H .
$$

If $\left\{\phi_{i}(\cdot-k): 1 \leq i \leq r, k \in Z\right\}$ is a frame for $H$, then there exists a dual frame $\left\{\tilde{\phi}_{k}\right\}_{k \in Z}$ for $\left\{\phi_{i}(\cdot-k): 1 \leq i \leq r, k \in Z\right\}$ [12, Theorem 5.6.5].

For $f \in H$,we can write

$$
\begin{aligned}
E_{f} & =\left\{\omega \in R \mid G_{f}(\omega)>0\right\}, \\
G_{f}(\omega) & =\sum_{k \in Z}|\widehat{f}(\omega+2 \pi k)|^{2}, \\
X E_{f} & = \begin{cases}1, & t \in E_{f}, \\
0, & t \notin E_{f} .\end{cases}
\end{aligned}
$$

Let

$$
\begin{aligned}
& \Phi=\left[\phi_{1}, \phi_{2}, \ldots, \phi_{r}\right]^{T}, \\
& \widetilde{\Phi}=\left[\tilde{\phi}_{1}, \tilde{\phi}_{2}, \ldots, \tilde{\phi}_{r}\right]^{T} .
\end{aligned}
$$


The reproducing kernel is defined as

$$
\begin{aligned}
q(t, \cdot) & =\sum_{l=1}^{r} \sum_{k \in Z} \overline{\phi_{l}(t-k)} \tilde{\phi}_{l}(\cdot-k) \\
& =\sum_{k \in Z} \Phi^{*}(t-k) \widetilde{\Phi}(\cdot-k)
\end{aligned}
$$

where ${ }^{*}$ denotes the Hermitian conjugate. Put $t=n+a_{m}$. Then

$$
\begin{aligned}
q\left(n+a_{m}, \cdot\right) & =\sum_{l=1}^{r} \sum_{k \in Z} \overline{\phi_{l}\left(n+a_{m}-k\right)} \tilde{\phi}_{l}(\cdot-k) \\
& =\sum_{k \in Z} \Phi^{*}\left(n+a_{m}-k\right) \widetilde{\Phi}(\cdot-k)
\end{aligned}
$$

where $1 \leq m \leq r, n \in Z, a_{m} \in[0,1)$ is constant, and $a_{i} \neq a_{j}$ for $i \neq j, i, j=1,2, \ldots, r$.

Let $A \subset R, \operatorname{Max}\{A\}$ means the largest number in subset $A$, and $\operatorname{Min}\{A\}$ means the smallest number in subset $A$.

For $f, g \in L^{2}(R)$, let bracket function $[f, g]$ be the function defined a.e. by $[f, g](\xi)=$ $\sum_{k \in Z} f(\xi+k) \overline{g(\xi+k)}$.

\section{General Uniform Sampling Theorem}

The main purpose of this section is to study the regular sampling theorem for frame in multiwavelet subspace.

Firstly, we start with some useful lemmas.

Lemma 3.1. Let $f, g \in L^{2}(R)$. If $\langle f\rangle \perp\langle g\rangle$ and $|h|,|s| \in\langle f\rangle \oplus\langle g\rangle$ for all $s \in\langle f\rangle, h \in\langle g\rangle$, then $[s, h]=0$ a.e.

Proof. Suppose that $\{f(\cdot-k)\}$ is a frame sequence in $L^{2}(R)$. Let $s \in\langle f\rangle$, then there exists a sequence $\left\{c_{i}\right\}_{i \in Z} \in l^{2}$ such that $s=\sum_{i \in Z} c_{i} f(\cdot-i)$.

Define

$$
x(s)= \begin{cases}1, & s \geq 0 \\ -1, & s<0 .\end{cases}
$$

We have

$$
\begin{aligned}
|s| & =\chi(s) s \\
& =\chi(s) \sum_{i \in Z} c_{i} f(\cdot-i) \\
& =\sum_{i \in Z} \chi(s) c_{i} f(\cdot-i) .
\end{aligned}
$$


It is easy to check that $\left\{\chi(s) c_{i}\right\}_{i \in Z} \in l^{2}$ and $|s| \perp\langle g\rangle$; thus $|s| \in\langle f\rangle$. Similar to the above argument, for all $h \in\langle g\rangle$, we get $|h| \in\langle g\rangle$.

Suppose that $\langle f\rangle \perp\langle g\rangle$ holds; by $|s| \in\langle f\rangle$ and $|h| \in\langle g\rangle$, we can obtain

$$
\begin{aligned}
0 & \leq \int_{T}\left|\sum_{k \in Z} s(x+k) \cdot \overline{h(x+k)}\right| d x \\
& \leq \int_{T} \sum_{k \in Z}|s(x+k) \cdot \overline{h(x+k)}| d x \\
& =\int_{R}|s(x) \cdot \overline{h(x)}| d x \\
& \leq\langle|s|,|h|\rangle \\
& =0 .
\end{aligned}
$$

It implies that $[s, h]=0$ a.e. The proof is completed.

Lemma 3.2. Let $\left\{f_{i}\right\}_{i \in\{1,2, \ldots, r\}} \subset L^{2}(R)$, then the following two assertions are equivalent:

(a) for any $\left\{c_{k}^{i}\right\}_{k \in Z} \in l^{2}, i \in\{1,2, \ldots, r\}, \sum_{i=1}^{r} \sum_{k \in Z} c_{k}^{i} f_{i}(x-k)$ converges pointwise to a continuous function;

(b) $\left\{f_{i}\right\}_{i \in\{1,2, \ldots, r\}} \subset C(R)$ and $\sup _{x} \sum_{i=1}^{r} \sum_{k \in Z}\left|f_{i}(x-k)\right|^{2}<+\infty$.

Proof. (a) $\Rightarrow$ (b). It is easy to see that for all $i \in\{1,2, \ldots, r\}, f_{i} \in C(R)$. For each $x \in R$, since $\sum_{i=1}^{r} \sum_{k \in Z} c_{k}^{i} f_{i}(x-k)$ is convergent for each $\left\{c_{k}^{i}\right\} \in l^{2}$, clearly, $\sum_{k \in Z}\left|f_{i}(x-k)\right|^{2}<\infty$. For each $x \in[0,1]$, define

$$
T_{x} c=\sum_{i=1}^{r} \sum_{k \in Z} c_{k}^{i} f_{i}(x-k), \quad \forall c=\left\{c_{k}^{i}\right\} \in l^{2}
$$

Then $T_{x}$ is a bounded linear functional on $l^{2}$ with the norm $\left\|T_{x}\right\|=\left(\sum_{i=1}^{r} \sum_{k \in Z}\left|f_{i}(x-k)\right|^{2}\right)^{1 / 2}$. For any $\left\{c_{k}^{i}\right\} \in l^{2}$, define $g(t)=\sum_{i=1}^{r} \sum_{k \in Z} c_{k}^{i} f_{i}(t-k)$. Since $g(t)$ is continuous on $R$, we have

$$
\sup _{x \in[0,1]}\left|T_{x} c\right|=\sup _{x \in[0,1]}|g(x)|<+\infty .
$$

By the Banach-Steinhaus theorem [13], $\sup _{x \in[0,1]}\left\|T_{x}\right\|<+\infty$, that is, $\sum_{i=1}^{r} \sum_{k \in Z}\left|f_{i}(x-k)\right|^{2}$ is bounded on $R$.

$(\mathrm{a}) \Rightarrow(\mathrm{b})$. By the Cauchy inequality, $\sum_{i=1}^{r} \sum_{k \in Z} c_{k}^{i} f_{i}(x-k)$ in convergent uniformly on $R$, so the limit function is continuous.

Lemma 3.3. Let $\left\{\phi_{i}(\cdot-k): 1 \leq i \leq r, k \in Z\right\}$ be a frame for $V_{0}$ with bounds $A$ and $B$. If $\left\langle\phi_{i}\right\rangle \perp\left\langle\phi_{j}\right\rangle$ for all $i, j \in\{1,2, \ldots, r\}, i \neq j$, then $\left\{\phi_{i}(\cdot-k)\right\}_{k \in Z}$ is a frame for the subspace $\left\langle\phi_{i}\right\rangle$. 
Proof. Suppose that $\left\{\phi_{i}(\cdot-k): 1 \leq i \leq r, k \in Z\right\}$ is a frame for the subspace $V_{0}$, then there exist constants $0<A \leq B<\infty$ such that

$$
A\|f\|^{2} \leq \sum_{i=1}^{r} k_{k \in Z} \sum\left|\left\langle f, \phi_{i}(x-k)\right\rangle\right|^{2} \leq \mathrm{B}\|f\|^{2} .
$$

Notice that $\left\langle\phi_{i}\right\rangle \perp\left\langle\phi_{j}\right\rangle$ for all $i, j \in\{1,2, \ldots, r\}, i \neq j$; if $f \in\left\langle\phi_{i}\right\rangle$, then $\left\langle f, \phi_{j}(x-k)\right\rangle=0$. Hence,

$$
A\|f\|^{2} \leq \sum_{k \in Z}\left|\left\langle f, \phi_{i}(x-k)\right\rangle\right|^{2} \leq \mathrm{B}\|f\|^{2} .
$$

By the definition of Frame, $\left\{\phi_{i}(\cdot-k)\right\}_{k \in Z}$ is a frame for the closed subspace $\left\langle\phi_{i}\right\rangle$. Then we get the desired result.

Lemma 3.4. Let $f \in L^{2}(R)$ and $f \in C(R)$, then there exists a set $E \in R,|R \backslash E|=0$ such that for any $x \in E$ and for all $\left\{c_{k}\right\}_{k \in Z} \in l^{2}, \sum_{k \in Z} c_{k} f(x-k)$ converges pointwise to a continuous function.

Proof. By $f \in L^{2}(R)$, then $\int_{T} \sum_{k \in Z}|f(x-k)|^{2} d x<\infty$. Hence there exists a set $E \in R,|R \backslash E|=0$ such that $\sup _{x \in E} \sum_{k \in Z}|f(x-k)|^{2}<\infty$. Notice that $f \in C(R)$; using Lemma 3.2, clearly, for any $\left\{c_{k}\right\}_{k \in Z} \in l^{2}, \sum_{k} c_{k} f(x-k)$ converges pointwise to a continuous function in set $E$.

From the above lemmas, we have the following result.

Theorem 3.5. Let $\left\{\phi_{i}(\cdot-k): 1 \leq i \leq r, k \in Z\right\}$ be a frame for $V_{0}$. Suppose that $\phi_{i}, 1 \leq i \leq r$ are continuous functions, $\left\langle\phi_{i}\right\rangle \perp\left\langle\phi_{j}\right\rangle$ for all $i \neq j, i, j \in\{1,2, \ldots, r\}$, and

$$
\sup _{x \in R} \sum_{i=1}^{r} \sum_{k \in Z}\left|\phi_{i}(x-k)\right|^{2}<+\infty
$$

Then

$$
f(t)=\int_{R} f(s) q(t, s) d s
$$

holds for each $f \in V_{0}$ and $t \in R$.

Proof. Suppose that $\left\{\phi_{i}(\cdot-k): 1 \leq i \leq r, k \in Z\right\}$ is a frame for $V_{0}$ and $\left\langle\phi_{i}\right\rangle \perp\left\langle\phi_{j}\right\rangle, i \neq j$, then, by [14, Proposition 3.1] and Lemma 3.3, there exist constants $0<A_{i} \leq B_{i}<\infty$ such that

$$
A_{i} X_{E_{\phi_{i}}}(\omega) \leq \sum_{n \in Z}\left|\widehat{\phi}_{i}(\omega+2 n \pi)\right|^{2} \leq B_{i} X_{E_{\phi_{i}}}(\omega), \quad \text { a.e. }
$$


From Lemma 4 in [15], we have the dual frame $\tilde{\phi}_{i}$ for $\left\{\phi_{i}(\cdot-k): 1 \leq i \leq r, k \in Z\right\}$, where $\tilde{\phi}_{i}$ is defined by

$$
\widetilde{\phi}_{i}(\omega)= \begin{cases}\frac{\widehat{\phi}_{i}(\omega)}{G_{\phi_{i}}(\omega)}, & \omega \in E_{\phi_{i}}, \\ 0, & \omega \notin E_{\phi_{i}} .\end{cases}
$$

Hence, for all $1 \leq i \leq r$,

$$
\frac{\widehat{\phi}_{i}(\omega)}{B_{i}} \leq \widehat{\widetilde{\phi}}_{i}(\omega) \leq \frac{\widehat{\phi}_{i}(\omega)}{A_{i}}
$$

holds.

So, it follows that

$$
\begin{aligned}
& \tilde{\phi}_{i}(x)=\int_{\omega \in R} \widehat{\widetilde{\phi}}_{i}(\omega) e^{i \omega x} d \omega \leq \frac{1}{A_{i}} \int_{\omega \in R} \widehat{\phi}_{i}(\omega) e^{i \omega x} d \omega=\frac{1}{A_{i}} \widehat{\phi}_{i}(x), \\
& \tilde{\phi}_{i}(x)=\int_{\omega \in R} \widehat{\widetilde{\phi}}_{i}(\omega) e^{i \omega x} d \omega \geq \frac{1}{B_{i}} \int_{\omega \in R} \widehat{\phi}_{i}(\omega) e^{i \omega x} d \omega=\frac{1}{B_{i}} \widehat{\phi}_{i}(x) .
\end{aligned}
$$

From above results, obviously, if

$$
\sup _{x \in R} \sum_{i=1}^{r} \sum_{k \in Z}\left|\phi_{i}(x-k)\right|^{2}<+\infty,
$$

then

$$
\sup _{x \in R} \sum_{i=1}^{r} \sum_{k \in Z}\left|\tilde{\phi}_{i}(x-k)\right|^{2}<+\infty
$$

Hence,

$$
\begin{aligned}
q(t, s) & =\sum_{l=1}^{r} \sum_{k \in Z} \overline{\phi_{l}(t-k)}, \tilde{\phi}_{l}(s-k) \\
& =\sum_{k \in Z} \Phi^{*}(t-k) \tilde{\Phi}(s-k)
\end{aligned}
$$

converges to $q(t, \cdot)$ in $L^{2}(R)$ for any $f \in V_{0}$ and $t \in R$. 
By $\left\langle\phi_{i}\right\rangle \perp\left\langle\phi_{j}\right\rangle$ for all $i, j \in\{1,2, \ldots, r\}, i \neq j$, it is easy to check that $\left\{\tilde{\phi}_{i}(\cdot-k): 1 \leq i \leq\right.$ $r, k \in Z\}$ is a dual frame for $\left\{\phi_{i}(\cdot-k): 1 \leq i \leq r, k \in Z\right\}$; then we have

$$
\begin{aligned}
f(t) & =\sum_{l=1}^{r} \sum_{k \in Z}\left\langle f(s), \tilde{\phi}_{l}(s-k)\right\rangle \phi_{l}(t-k) \\
& =\left\langle f(s), \sum_{l=1}^{r} \sum_{k \in Z} \overline{\phi_{l}(t-k)}, \tilde{\phi}_{l}(s-k)\right\rangle \\
& =\left\langle f(s), \sum_{k \in Z} \Phi^{*}(t-k) \widetilde{\Phi}(s-k)\right\rangle \\
& =\int_{R} f(s) q(t, s) d s
\end{aligned}
$$

for any $f \in V_{0}$ and $t \in R$. So we get the desired result.

Based on these facts, the following sampling theorem is established.

Theorem 3.6. Let $\left\{\phi_{i}(\cdot-k): 1 \leq i \leq r, k \in Z\right\}$ be a frame for $V_{0}$. Suppose that $\left\langle\phi_{i}\right\rangle \perp\left\langle\phi_{j}\right\rangle$ for all $i, j \in\{1,2, \ldots, r\}, i \neq j$, and $\phi_{i}, 1 \leq i \leq r$ are continuous such that

$$
\sup _{t \in R} \sum_{i=1}^{r} \sum_{k \in Z}\left|\phi_{i}(t-k)\right|^{2}<+\infty
$$

If there exist constants $A_{i}$ and $B_{i}, 0<A_{i} \leq B_{i}<+\infty$ such that

$$
A_{i} \chi_{E_{\phi_{i}}}(\omega) \leq\left|\widehat{\phi}_{i}^{*}(\omega)\right| \leq B_{i} \chi_{E_{\phi_{i}}}(\omega), \quad \text { a.e. } \omega \in R,
$$

where $\widehat{\phi}_{i}^{*}(\omega)=\sum_{n \in Z} \phi_{i}(n) e^{-i n \omega}$, then there exists a frame $\left\{s_{i}(\cdot-k): 1 \leq i \leq r, k \in Z\right\}$ of $V_{0}$, for $i, j \in\{1,2, \ldots, r\}, i \neq j$

$$
f(t)=\sum_{n \in Z} \sum_{m=1}^{r} f\left(n+a_{m}\right) s_{m}(t-n), \quad f \in V_{0}
$$

holds, where the convergence is in $L^{2}(R)$.

Proof. Define the function $q_{i}(t, s)$ by $q_{i}(t, s)=\sum_{k \in Z} \overline{\phi_{i}(t-k)} \tilde{\phi}_{i}(s-k)$, where $\tilde{\phi}_{i}$ is defined by (3.11). From Lemma 3.3, it is easy to see that $\left\{\phi_{i}(\cdot-k)\right\}_{k \in Z}$ and $\left\{\tilde{\phi}_{i}(\cdot-k)\right\}_{k \in Z}$ are the frames for the subspace $\left\langle\phi_{i}\right\rangle$.

Notice that

$$
\begin{gathered}
\widehat{\phi}_{i}(n, \omega)=\overline{\widehat{\phi}_{i}^{*}(\omega)} \widehat{\tilde{\phi}}_{i}(\omega), \\
A_{i} X_{E_{\phi_{i}}}(\omega) \leq\left|\widehat{\phi}_{i}^{*}(\omega)\right| \leq B_{i} X_{E_{\phi_{i}}}(\omega), \quad \text { a.e. } \omega \in R .
\end{gathered}
$$


From the proof process of Proposition 3.1 in [14], $\left\{q_{i}(n, \cdot)\right\}_{n \in Z}=\left\{q_{i}(0, \cdot-n)\right\}_{n \in Z}$ is a frame for the subspace $\left\langle\phi_{i}\right\rangle$. Similar to the above argument, obviously, for $1 \leq m \leq r,\left\{q_{i}\left(n+a_{m}, \cdot\right)\right\}_{n \in Z}=$ $\left\{q_{i}\left(0, \cdot-n-a_{m}\right)\right\}_{n \in Z}$ is the frame for the subspace $\left\langle\phi_{i}\right\rangle$. Hence, for all $f_{i} \in V_{i}$, for all $m \in$ $\{1,2, \ldots, r\}$, there exist constants $0<C_{i}^{m} \leq D_{i}^{m}<\infty$ such that

$$
C_{i}^{m}\left\|f_{i}\right\|^{2} \leq \sum_{n \in Z}\left|\left\langle f_{i}, q_{i}\left(n+a_{m}, \cdot\right)\right\rangle\right|^{2} \leq D_{i}^{m}\left\|f_{i}\right\|^{2}
$$

Thus, we have

$$
f_{i}(s)=\sum_{n \in Z}\left\langle f_{i}(s), q_{i}\left(n+a_{m}, s\right)\right\rangle \tilde{q}_{i}\left(n+a_{m}, s\right),
$$

where $\tilde{q}_{i}\left(n+a_{m}, s\right)$ is the dual frame for $q_{i}\left(n+a_{m}, s\right)$.

From above results, we get

$$
\sum_{i=1}^{r} C_{i}^{m}\left\|f_{i}\right\|^{2} \leq \sum_{i=1}^{r} \sum_{n \in Z}\left|\left\langle f_{i}, q_{i}\left(n+a_{m}, s\right)\right\rangle\right|^{2} \leq \sum_{i=1}^{r} D_{i}^{m}\left\|f_{i}\right\|^{2},
$$

where $m \in\{1,2, \ldots, r\}$.

Notice that $\left\{\phi_{i}(\cdot-k)\right\}_{k \in Z},\left\{q_{i}\left(0, \cdot-n-a_{m}\right)\right\}_{n \in Z, m \in\{1,2, \ldots, r\}}$ and $\left\{q_{i}(0, \cdot-n)\right\}_{n \in Z}$ are the frame for subspace $\left\langle\phi_{i}\right\rangle$; by [16, Lemma 3], we have $E_{\phi_{i}}=E_{q_{i}\left(0,-, n-a_{m}\right)}=E_{q_{i}(0,-n)}$ (except a null measurable set).

By Lemma 3.3, it follows that for all $f \in V_{0}, f=\sum_{i=1}^{r} f_{i}$ holds, where $f_{i} \in V_{i}$. Again by Lemma 3.3, we have $V_{i} \perp V_{l}, 1 \leq i \neq l \leq r$, then,

$$
\|f\|^{2}=\left\langle\sum_{i=1}^{r} f_{i}, \overline{\sum_{l=1}^{r} f_{l}}\right\rangle=\sum_{i=1}^{r} \sum_{l=1}^{r}\left\langle f_{i}, \overline{f_{l}}\right\rangle=\sum_{i=1}^{r}\left\langle f_{i}, \overline{f_{i}}\right\rangle=\sum_{i=1}^{r}\left\|f_{i}\right\|^{2} .
$$

From (3.23) and (3.25), for all $m \in\{1,2, \ldots, r\}$, we get

$$
\begin{aligned}
\operatorname{Min}\left\{C_{i}^{m}\right\}_{i=1}^{r}\|f\|^{2} & =\operatorname{Min}\left\{C_{i}^{m}\right\}_{i=1}^{r} \sum_{i=1}^{r}\left\|f_{i}\right\|^{2} \\
& \leq \sum_{i=1}^{r} C_{i}^{m}\left\|f_{i}\right\|^{2} \leq \sum_{i=1}^{r} \sum_{n \in Z}\left|\left\langle f_{i}, q_{i}\left(n+a_{m},\right)\right\rangle\right|^{2}, \\
\sum_{i=1}^{r} \sum_{n \in Z}\left|\left\langle f_{i}, q_{i}\left(n+a_{m}, \cdot\right)\right\rangle\right|^{2} & \leq \sum_{i=1}^{r} D_{i}^{m}\left\|f_{i}\right\|^{2} \\
& \leq \operatorname{Max}\left\{D_{i}^{m}\right\}_{i=1}^{r} \sum_{i=1}^{r}\left\|f_{i}\right\|^{2}=\operatorname{Max}\left\{D_{i}^{m}\right\}_{i=1}^{r}\|f\|^{2} .
\end{aligned}
$$


For

$$
\begin{aligned}
\left\langle f(t), q_{i}\left(n+a_{i}, t\right)\right\rangle & =\int_{R} \sum_{l=1}^{r} f_{l}(t) \overline{q_{i}\left(n+a_{i}, t\right)} d t \\
& =\sum_{l=1}^{r} \int_{R} f_{l}(t) \overline{q_{i}\left(n+a_{i}, t\right)} d t \\
& =\int_{R} f_{i}(t) \overline{q_{i}\left(n+a_{i}, t\right)} d t \\
& =\left\langle f_{i}(t), q_{i}\left(n+a_{i}, t\right)\right\rangle,
\end{aligned}
$$

notice (3.26), we have

$$
\begin{aligned}
\operatorname{Min}\left\{C_{i}^{i}\right\}_{i=1}^{r}\|f\|^{2} & =\operatorname{Min}\left\{C_{i}^{i}\right\}_{i=1}^{r} \sum_{i=1}^{r}\left\|f_{i}\right\|^{2} \\
& \leq \sum_{i=1}^{r} C_{i}^{i}\left\|f_{i}\right\|^{2} \\
& \leq \sum_{n \in Z} \sum_{i=1}^{r}\left|\left\langle f_{i}, q_{i}\left(n+a_{i}, \cdot\right)\right\rangle\right|^{2} \\
& =\sum_{n \in Z} \sum_{i=1}^{r}\left|\left\langle f, q_{i}\left(n+a_{i}, \cdot\right)\right\rangle\right|^{2}, \\
\sum_{n \in Z} \sum_{i=1}^{r}\left|\left\langle f, q_{i}\left(n+a_{i}, \cdot\right)\right\rangle\right|^{2} & =\sum_{n \in Z} \sum_{i=1}^{r}\left|\left\langle f_{i}, q_{i}\left(n+a_{i}, \cdot\right)\right\rangle\right|^{2} \\
& \leq \sum_{i=1}^{r} D_{i}^{i}\left\|f_{i}\right\|^{2} \\
& \leq \operatorname{Max}\left\{D_{i}^{i}\right\}_{i=1}^{r} \sum_{i=1}^{r}\left\|f_{i}\right\|^{2} \\
& =\operatorname{Max}\left\{D_{i}^{i}\right\}_{i=1}^{r}\|f\|^{2} .
\end{aligned}
$$

Let $C=\operatorname{Min}\left\{C_{i}^{i}\right\}_{i=1}^{r}, D=\operatorname{Max}\left\{D_{i}^{i}\right\}_{i=1}^{r}$, then for all $f \in V_{0}$, there exist constants number $0<C \leq D<\infty$ such that

$$
C\|f\|^{2} \leq \sum_{i=1}^{r} \sum_{n \in Z}\left|\left\langle f, q_{i}\left(n+a_{i}, \cdot\right)\right\rangle\right|^{2} \leq D\|f\|^{2} .
$$

Hence, according to the definition of frame, $\left\{q_{i}\left(n+a_{i}, s\right): 1 \leq i \leq r, n \in Z\right\}$ is the frame for the subspace $V_{0}$. Let $q\left(n+a_{i}, s\right)=\sum_{l=1}^{r} q_{l}\left(n+a_{i}, s\right)$; by Theorem 3.5, clearly, $\left\langle f(s), q\left(n+a_{i}, s\right)\right\rangle=$ $f\left(n+a_{i}\right)$. 
Notice that

$$
\begin{aligned}
\left|\left\langle f(s), \sum_{l=1}^{r} q_{l}\left(n+a_{i}, s\right)\right\rangle\right|^{2} & =\left|\sum_{l=1}^{r}\left\langle f(s), q_{l}\left(n+a_{i}, s\right)\right\rangle\right|^{2} \\
& \leq \sum_{l=1}^{r}\left|\left\langle f(s), q_{l}\left(n+a_{i}, s\right)\right\rangle\right|^{2} .
\end{aligned}
$$

Using (3.27) and (3.22), then

$$
\begin{aligned}
\sum_{i=1}^{r} \sum_{n \in Z}\left|\left\langle f(s), q\left(n+a_{i}, s\right)\right\rangle\right|^{2} & =\sum_{i=1}^{r} \sum_{n \in Z}\left|\left\langle f(s), \sum_{l=1}^{r} q_{l}\left(n+a_{i}, s\right)\right\rangle\right|^{2} \\
& \leq \sum_{i=1}^{r} \sum_{n \in Z} \sum_{l=1}^{r}\left|\left\langle f(s), q_{l}\left(n+a_{i}, s\right)\right\rangle\right|^{2} \\
& =\sum_{i=1}^{r} \sum_{n \in Z} \sum_{l=1}^{r}\left|\left\langle f_{l}(s), q_{l}\left(n+a_{i}, s\right)\right\rangle\right|^{2} \\
& \leq \sum_{l=1}^{r}\left(\sum_{i=1}^{r} D_{l}^{i}\left\|f_{l}\right\|^{2}\right) \\
& \leq \sum_{l=1}^{r} \operatorname{Max}\left\{D_{l}^{i}: i \in\{1,2, \ldots, r\}\right\}\left\|f_{l}\right\|^{2} \\
& \leq \operatorname{Max}\left\{\operatorname{Max}\left\{D_{l}^{i}: i \in\{1,2, \ldots, r\}\right\}: l \in\{1,2, \ldots, r\}\right\}\|f\|^{2} \\
& =D^{\prime}\|f\|^{2} .
\end{aligned}
$$

For $f_{l} \in\left\langle\phi_{l}\right\rangle, f_{p} \in\left\langle\phi_{p}\right\rangle$, by Lemmas 3.1 and 3.4, notice that for all $l \in\{1,2, \ldots, r\}$, $\left\{f_{l}\left(n+a_{i}\right)\right\}_{n \in Z} \in l^{2}$, then $\sum_{n \in Z} f_{l}\left(n+a_{i}\right) \overline{f_{p}\left(n+a_{i}\right)}=0$, for all $i \in\{1,2, \ldots, r\}, l \neq p$. Hence,

$$
\begin{aligned}
\sum_{i=1}^{r} \sum_{n \in Z}\left|\left\langle f, q\left(n+a_{i}, \cdot\right)\right\rangle\right|^{2} & =\sum_{i=1}^{r} \sum_{n \in Z}\left(\left\langle f, q\left(n+a_{i}, \cdot\right)\right\rangle\right) \overline{\left(\left\langle f, q\left(n+a_{i}, \cdot\right)\right\rangle\right)} \\
& =\sum_{i=1}^{r} \sum_{n \in Z}\left(\sum_{l=1}^{r}\left\langle f_{l}, q_{l}\left(n+a_{i}, \cdot\right)\right\rangle\right)\left(\overline{\sum_{p=1}^{r}\left\langle f_{p}, q_{p}\left(n+a_{i}, \cdot\right)\right\rangle}\right) \\
& =\sum_{i=1}^{r} \sum_{n \in Z} \sum_{l=1}^{r}\left|\left\langle f_{l}, q_{l}\left(n+a_{i}, \cdot\right)\right\rangle\right|^{2} \\
& \geq \operatorname{Min}\left\{\operatorname{Min}\left\{C_{l}^{i}: i \in\{1,2, \ldots, r\}\right\}: l \in\{1,2, \ldots, r\}\right\}\|f\|^{2} \\
& \geq C^{\prime}\|f\|^{2}
\end{aligned}
$$


holds. From (3.31) and (3.32), we get that $\left\{q\left(n+a_{i}, s\right): i=1,2, \ldots, r, n \in Z\right\}$ is the frame for the subspace $V_{0}$. Then there exists a dual frame $\left\{s_{i}(\cdot-k): 1 \leq i \leq r, k \in Z\right\}$ of $\left\{q\left(n+a_{i}, s\right)\right.$ : $i=1,2, \ldots, r, n \in Z\}$ such that

$$
f=\sum_{i=1}^{r} \sum_{n \in Z}\left\langle f, q\left(n+a_{i}, \cdot\right)\right\rangle s_{i}(\cdot-k)=\sum_{i=1}^{r} \sum_{n \in Z} f\left(a_{i}+n\right) s_{i}(\cdot-k), \quad \forall f \in V_{0} .
$$

The proof is completed.

\section{General Irregular Sampling Theorem}

In many realistic situations, we try to reconstruct a continuous signal $f \in V_{0}$ by using its discrete samples $\left\{f\left(n+a_{m}+\delta_{m}^{n}\right): 1 \leq m \leq r, n \in Z\right\}$. Obviously, the samples cannot be arbitrary; we must discuss the values $\delta_{m}^{n}$ of deviation from the uniformly noninteger points. The following theorem studies this problem.

Based on the results in Section 3, we get the irregular sampling theorems.

Theorem 4.1. Let $\left\{\phi_{i}(\cdot-k): 1 \leq i \leq r, k \in Z\right\}$ be a frame for $V_{0}$ with bounds $A$ and $B$. Suppose that $\left\langle\phi_{i}\right\rangle \perp\left\langle\phi_{j}\right\rangle$ for all $i, j \in\{1,2, \ldots, r\}, i \neq j$, and $\phi_{i}, 1 \leq i \leq r$ are continuous such that

$$
\sup _{t \in R} \sum_{i=1}^{r} \sum_{k \in Z}\left|\phi_{i}(t-k)\right|^{2}<+\infty
$$

Moreover, suppose that there exist constants $A_{i}$ and $B_{i}, 0<A_{i} \leq B_{i}<+\infty$ such that

$$
A_{i} \chi_{E_{\phi_{i}}}(\omega) \leq\left|\widehat{\phi}_{i}^{*}(\omega)\right| \leq B_{i} \chi_{E_{\phi_{i}}}(\omega) \text {, a.e. } \omega \in R \text {. }
$$

If there exists a sequence $\left\{\delta_{m}^{n}: n \in Z, m=1,2, \ldots, r\right\}$ and two constants $C$ and $D, 0<C \leq D<\infty$ such that

$$
C \sum_{m=1}^{r} \sum_{n \in Z}\left|f\left(n+a_{m}\right)\right|^{2} \leq \sum_{m=1}^{r} \sum_{n \in Z}\left|f\left(n+a_{m}+\delta_{m}^{n}\right)-f\left(n+a_{m}\right)\right|^{2} \leq D \sum_{m=1}^{r} \sum_{n \in Z}\left|f\left(n+a_{m}\right)\right|^{2}
$$

holds for any $f \in V_{0}$. Then, there exists a frame $\left\{s_{m, n}(t): 1 \leq m \leq r, n \in Z\right\}$ for $V_{0}$ such that for any $f \in V_{0}$,

$$
f(t)=\sum_{n \in Z} \sum_{m=1}^{r} f\left(n+a_{m}+\delta_{m}^{n}\right) s_{m, n}(t)
$$

holds, where the convergence is both in $L^{2}(R)$ and uniform on $R$.

Proof. From the argument of Theorem 3.6, it is easy to see that $\left\{q\left(a_{1}, \cdot-n\right), q\left(a_{2}, \cdot-n\right)\right.$, $\left.\ldots, q\left(a_{r}, \cdot-n\right)\right\}_{n \in Z}=\left\{q\left(n+a_{1}, \cdot\right), q\left(n+a_{2}, \cdot\right), \ldots, q\left(n+a_{r}, \cdot\right)\right\}_{n \in Z}$ is a frame for the closed 
subspace $V_{0}$ of $L^{2}(R)$. Hence, there exist two constants $C_{1}$ and $C_{2}, 0<C_{1} \leq C_{2}<\infty$ such that

$$
C_{1}\|f\|^{2} \leq \sum_{n \in Z} \sum_{k=l}^{r}\left|\left\langle f, q\left(n+a_{k}, \cdot\right)\right\rangle\right|^{2}=\sum_{n \in Z} \sum_{k=l}^{r}\left|f\left(n+a_{k}\right)\right|^{2} \leq C_{2}\|f\|^{2}, \quad \forall f \in V_{0} .
$$

By (4.3), we have

$$
C C_{1}\|f\|^{2} \leq \sum_{n \in Z} \sum_{k=l}^{r}\left|\left\langle f, q\left(n+a_{k}+\delta_{k}^{n} \cdot\right)\right\rangle\right|^{2}=\sum_{n \in Z} \sum_{k=l}^{r}\left|f\left(n+a_{k}+\delta_{k}^{n}\right)\right|^{2} \leq D C_{2}\|f\|^{2} .
$$

Then, $\left\{q\left(n+a_{1}+\delta_{1}^{n}, \cdot\right), q\left(n+a_{2}+\delta_{2}^{n}, \cdot\right), \ldots, q\left(n+a_{r}+\delta_{r}^{n}, \cdot\right)\right\}_{n}$ is also a frame for the closed subspace $V_{0}$ of $L^{2}(R)$. So, there exists a dual frame $\left\{s_{i}(\cdot-k): 1 \leq i \leq r, k \in Z\right\}$ of $\left\{q\left(n+a_{i}+\delta_{i}^{n}, \cdot\right): 1 \leq i \leq r, k \in Z\right\}$ such that

$$
f(t)=\sum_{n \in Z} \sum_{m=1}^{r} f\left(n+a_{m}+\delta_{m}^{n}\right) s_{m}(t-n)
$$

for any $f \in V_{0}$.

Theorem 4.2. Let $\left\{\phi_{i}(\cdot-k): 1 \leq i \leq r, k \in Z\right\}$ be a frame for $V_{0}$ with bounds $A$ and B. Suppose that $\left\langle\phi_{i}\right\rangle \perp\left\langle\phi_{j}\right\rangle$ for all $i, j \in\{1,2, \ldots, r\}, i \neq j$, and $\phi_{i}, 1 \leq i \leq r$ are continuous such that

$$
\sup _{t \in R} \sum_{i=1}^{r} \sum_{k \in Z}\left|\phi_{i}(t-k)\right|^{2}<+\infty
$$

Moreover, suppose that there exist constants $A_{i}$ and $B_{i}, 0<A_{i} \leq B_{i}<+\infty$ such that

$$
A_{i} X_{E_{\phi_{i}}}(\omega) \leq\left|\widehat{\phi}_{i}^{*}(\omega)\right| \leq B_{i} \chi_{E_{\phi_{i}}}(\omega), \quad \text { a.e. } \omega \in R \text {. }
$$

If there exists a sequence $\left\{\delta_{m}^{n}: n \in Z, m=1,2, \ldots, r\right\}$ and a constant $0<\theta<1$ such that

$$
\sum_{m=1}^{r} \sum_{n \in Z}\left|f\left(n+a_{m}+\delta_{m}^{n}\right)-f\left(n+a_{m}\right)\right|^{2} \leq \theta^{2} \sum_{m=1}^{r} \sum_{n \in Z}\left|f\left(n+a_{m}\right)\right|^{2}
$$

holds for any $f \in V_{0}$, then, there exists a frame $\left\{s_{m, n}(t): 1 \leq m \leq r, n \in Z\right\}$ for $V_{0}$ such that for any $f \in V_{0}$

$$
f(t)=\sum_{n \in Z} \sum_{m=1}^{r} f\left(n+a_{m}+\delta_{m}^{n}\right) s_{m, n}(t)
$$

holds, where the convergence is both in $L^{2}(R)$ and uniform on $R$. 
Proof. If

$$
\sum_{m=1}^{r} \sum_{n \in Z}\left|f\left(n+a_{m}+\delta_{m}^{n}\right)-f\left(n+a_{m}\right)\right|^{2} \leq \theta^{2} \sum_{m=1}^{r} \sum_{n \in Z}\left|f\left(n+a_{m}\right)\right|^{2},
$$

then

$$
\begin{aligned}
\left(1-\theta^{2}\right) \sum_{m=1}^{r} \sum_{n \in Z}\left|f\left(n+a_{m}\right)\right|^{2} & \leq \sum_{m=1}^{r} \sum_{n \in Z}\left|f\left(n+a_{m}+\delta_{m}^{n}\right)\right|^{2} \\
& \leq\left(1+\theta^{2}\right) \sum_{m=1}^{r} \sum_{n \in Z}\left|f\left(n+a_{m}\right)\right|^{2}
\end{aligned}
$$

By Theorem 4.1, we get the desired result.

\section{Perturbation of Uniform Noninteger Sampling in Shift-Invariant Spaces}

Our objective is to find explicit formulas or algorithms to calculate the ranges of the perturbations of uniform noninteger sampling in shift-invariant spaces. In order to establish the algorithm for perturbations of uniform noninteger sampling for frame in shift-invariant spaces, we need to introduce the function class $L_{a_{m}}^{\lambda}[a, b](\lambda>0, \sigma \in[0,1), 0 \in[a, b] \subset$ $[-1,1])$ defined and used in [10].

Definition 5.1. $L_{a_{m}}^{\lambda}[a, b](\lambda>0$ and $\sigma \in[0,1), 0 \in[a, b] \subset[-1,1])$ consists of all the measurable functions $\mathrm{f}$, for which the norm

$$
\|f\|_{L_{a_{m}}^{\lambda}[a, b]}=\sup _{\left\{r_{k}\right\}_{k} \subset[a, b]} \frac{\sum_{k}\left|f\left(k+\sigma+r_{k}\right)-f(k+\sigma)\right|}{\sup _{k}\left|r_{k}\right|^{\lambda}}<\infty .
$$

Theorem 5.2. Let $\left\{\phi_{i}(\cdot-k): 1 \leq i \leq r, k \in Z\right\}$ be a frame for $V_{0}$ with bounds $A$ and $B$. Suppose that there exist constants $A_{i}$ and $B_{i}, 0<A_{i} \leq B_{i}<+\infty$ such that

$$
A_{i} X_{E_{\phi_{i}}}(\omega) \leq\left|\hat{\phi}_{i}^{*}(\omega)\right| \leq B_{i} \chi_{E_{\phi_{i}}}(\omega) \text {, a.e. } \omega \in R
$$

Moreover, suppose that $\left\langle\phi_{i}\right\rangle \perp\left\langle\phi_{j}\right\rangle$ for all $i, j \in\{1,2, \ldots, r\}, i \neq j$, and $\phi_{i}, 1 \leq i \leq r$ are continuous such that

$$
\sup _{t \in R} \sum_{i=1}^{r} \sum_{k \in Z}\left|\phi_{i}(t-k)\right|^{2}<+\infty
$$

Then, for any $\left\{\delta_{m}^{n}\right\} \subset\left[-\theta_{\Phi}, \theta_{\Phi}\right] \cap[a, b], 1 \leq m \leq r, n \in Z$, there is a frame $\left\{S_{m, n}\right\}$ of $V_{0}$ such that

$$
f(t)=\sum_{n \in Z} \sum_{m=1}^{r} f\left(n+a_{m}+\delta_{m}^{n}\right) s_{m}(t-n)
$$


holds in $L^{2}(R)$ for any $f \in V_{0}$ if

$$
\delta_{\Phi}<\left(\frac{C^{1 / 2}}{\sum_{m=1}^{r}\left\|\phi_{m}\right\|_{L_{a_{m}}^{\lambda}[a, b]}}\right)^{1 / \lambda} .
$$

Proof. Notice that $\left\{\phi_{i}(\cdot-k): 1 \leq i \leq r, k \in Z\right\}$ is a frame for $V_{0}$, then, for all $f \in V_{0}$, there exists a square summable sequence $\left\{c_{m}^{n}\right\}$ such that $f=\sum_{n \in Z} \sum_{m=1}^{r} c_{m}^{n} \phi_{m}(\cdot-n)$ holds in $L^{2}(R)$.

Similar to the argument of Theorem 5 in [10], let

$$
\begin{aligned}
\Delta= & \sum_{n \in Z} \sum_{m=1}^{r}\left|f\left(n+a_{m}+\delta_{m}^{n}\right)-f\left(n+a_{m}\right)\right|^{2} \\
= & \sum_{n \in Z} \sum_{m=1}^{r}\left|\sum_{k \in Z} \sum_{i=1}^{r} c_{i}^{k}\left(\phi_{i}\left(n+a_{m}+\delta_{m}^{n}-k\right)-\phi_{i}\left(n+a_{m}-k\right)\right)\right|^{2} \\
= & \sum_{n \in Z} \sum_{m=1}^{r} \sum_{k \in Z} \sum_{i=1}^{r} \sum_{l \in Z} \sum_{j=1}^{r} c_{i}^{k} c_{j}^{l}\left(\phi_{i}\left(n+a_{m}+\delta_{m}^{n}-k\right)-\phi_{i}\left(n+a_{m}-k\right)\right) \\
& \times\left(\phi_{j}\left(n+a_{m}+\delta_{m}^{n}-k\right)-\phi_{j}\left(n+a_{m}-k\right)\right) \\
= & \sum_{k \in Z} \sum_{i=1}^{r} \sum_{l \in Z} \sum_{j=1}^{r} c_{i}^{k} c_{j}^{l} \sum_{n \in Z} \sum_{m=1}^{r}\left(\phi_{i}\left(n+a_{m}+\delta_{m}^{n}-k\right)-\phi_{i}\left(n+a_{m}-k\right)\right) \\
& \times\left(\phi_{j}\left(n+a_{m}+\delta_{m}^{n}-k\right)-\phi_{j}\left(n+a_{m}-k\right)\right) .
\end{aligned}
$$

Take

$$
\begin{aligned}
a_{k, i, l, j}= & \sum_{n \in Z} \sum_{m=1}^{r}\left(\phi_{i}\left(n+a_{m}+\delta_{m}^{n}-k\right)-\phi_{i}\left(n+a_{m}-k\right)\right) \\
& \times\left(\phi_{j}\left(n+a_{m}+\delta_{m}^{n}-k\right)-\phi_{j}\left(n+a_{m}-k\right)\right) .
\end{aligned}
$$

Then, $a_{k, i l, l, j}=a_{l, j ; k, i}$ holds for any $k, l \in Z, 1 \leq i, j \leq r$, and

$$
\begin{aligned}
\Delta & =\sum_{k \in Z} \sum_{i=1}^{r} \sum_{l \in Z} \sum_{j=1}^{r} c_{i}^{k} c_{j}^{l} a_{k, i ; l, j} \leq \sum_{k \in Z} \sum_{i=1}^{r} \sum_{l \in Z} \sum_{j=1}^{r}\left|a_{k, i i l, j}\right| \frac{\left(\left(c_{i}^{k}\right)^{2}+\left(c_{j}^{l}\right)^{2}\right)}{2} \\
& =\frac{1}{2}\left(\sum_{k \in Z} \sum_{i=1}^{r}\left(\sum_{l \in Z} \sum_{j=1}^{r}\left|a_{k, i, i, j}\right|\right)\left(c_{i}^{k}\right)^{2}+\sum_{l \in Z} \sum_{j=1}^{r}\left(\sum_{k \in Z} \sum_{i=1}^{r}\left|a_{k, i, l, j}\right|\right)\left(c_{j}^{l}\right)^{2}\right) \\
& =\sum_{k \in Z} \sum_{i=1}^{r}\left(\sum_{l \in Z} \sum_{j=1}^{r}\left|a_{k, i, l, j}\right|\right)\left(c_{i}^{k}\right)^{2} \leq\left(\sup _{k \in Z, 1 \leq i \leq r} \sum_{l \in Z} \sum_{j=1}^{r}\left|a_{k, i l, l, j}\right|\right) \sum_{k \in Z} \sum_{i=1}^{r}\left(c_{i}^{k}\right)^{2} .
\end{aligned}
$$


Moreover

$$
\begin{aligned}
\sup _{k \in Z, 1 \leq i \leq r} \sum_{l \in Z} \sum_{j=1}^{r}\left|a_{k, i, l, j}\right| \leq & \sup _{k \in Z, 1 \leq i \leq r} \sum_{l \in Z} \sum_{j=1}^{r} \sum_{n \in Z} \sum_{m=1}^{r}\left|\phi_{i}\left(n+a_{m}+\delta_{m}^{n}-k\right)-\phi_{i}\left(n+a_{m}-k\right)\right| \\
& \times\left|\phi_{j}\left(n+a_{m}+\delta_{m}^{n}-l\right)-\phi_{j}\left(n+a_{m}-l\right)\right| \\
= & \sup _{k \in Z, 1 \leq i \leq r} \sum_{n \in Z} \sum_{m=1}^{r}\left|\phi_{i}\left(n+a_{m}+\delta_{m}^{n}-k\right)-\phi_{i}\left(n+a_{m}-k\right)\right| \\
& \times \sum_{l \in Z} \sum_{j=1}^{r}\left|\phi_{j}\left(n+a_{m}+\delta_{m}^{n}-l\right)-\phi_{j}\left(n+a_{m}-l\right)\right| \\
\leq & \left(\sum_{m=1}^{r}\left\|\phi_{m}\right\|_{L_{a_{m}}^{\lambda}[a, b]}\right)^{2}\left(\sup _{n, m}\left|\delta_{m}^{n}\right|^{2}\right) .
\end{aligned}
$$

Hence

$$
\Delta \leq \sum_{k \in Z} \sum_{i=1}^{r}\left(c_{i}^{k}\right)^{2}\left(\sum_{m=1}^{r}\left\|\phi_{m}\right\|_{L_{a_{m}}^{\lambda}[a, b]}\right)^{2}\left(\sup _{n, m}\left|\delta_{m}^{n}\right|^{\lambda}\right)^{2} .
$$

From the argument in Theorem 3.6, $\left\{q\left(n+a_{i}, \cdot\right): n \in Z, 1 \leq i \leq r\right\}$ is a frame for the closed subspace $V_{0}$. By (3.25), then

$$
\sum_{i=1}^{r} \sum_{n \in Z}\left|f\left(n+a_{i}\right)\right|^{2}=\sum_{i=1}^{r} \sum_{n \in Z}\left|\left\langle f, q\left(n+a_{i}\right)\right\rangle\right|^{2} \geq C\|f\|^{2} \geq C \sum_{i=1}^{r}\|f\|_{i} .
$$

Again from the proof process in Theorem 3.6, obviously,

$$
\begin{aligned}
\left\|f_{i}\right\|^{2} & \geq \sum_{k \in Z}\left|\left\langle f_{i}, q_{i}\left(k+a_{i}, \cdot\right)\right\rangle\right|^{2} \\
& =\sum_{k \in Z}\left|f_{i}(k)\right|^{2} \\
& =\frac{1}{2 \pi}\left\|\sum_{k \in Z} \sum_{l \in Z} c_{i}^{l} \phi_{i}(k-l) e^{-i k \omega}\right\|_{*}^{2} \\
& =\frac{1}{2 \pi}\left\|\hat{\phi}_{i}^{*} \sum_{k \in Z} c_{i}^{k}\right\|_{*}^{2} \\
& \geq A_{i}^{2} \sum_{k \in Z}\left(c_{i}^{k}\right)^{2}
\end{aligned}
$$


holds, where $\|f\|_{*}$ is defined by

$$
\|f\|_{*}=\left(\int_{0}^{2 \pi}|f(t)|^{2}\right)^{1 / 2}
$$

then

$$
\sum_{i=1}^{r} \sum_{n \in Z}\left|f\left(n+a_{i}\right)\right|^{2} \geq C \operatorname{Min}\left\{A_{1}^{2}, A_{2}^{2}, \ldots, A_{r}^{2}\right\} \sum_{i=1}^{r} \sum_{k \in Z}\left(c_{i}^{k}\right)^{2}
$$

By Theorem 4.2, we only need to show that

$$
\left(\sum_{m=1}^{r}\left\|\phi_{m}\right\|_{L_{a_{m}}^{\lambda}[a, b]}\right)^{2}\left(\sup _{n, m}\left|\delta_{m}^{n}\right|^{\lambda}\right)^{2} \leq \theta^{2} C .
$$

This is exactly implied by (5.5). We get the desired result.

\section{Conclusion}

Multiwavelets have orthogonality, regularity, short compact support, symmetry, and high approximation order. It is not possible in the scalar wavelet case. In our paper, we study the sampling theorem for frames in multiwavelet subspaces and obtain the sufficient condition under which the regular sampling theorem holds. Notice that the measurements may not be made at uniform points; we establish an irregular sampling theorem for frame in multiwavelet subspaces. Finally, an estimate for the perturbations of regular sampling in shift-invariant spaces is derived.

\section{Acknowledgments}

This work was supported by the National Natural Science Foundation of China (no. 10971228) and the Henan Provincial Natural Science Foundation of China (no. 102300410205).

\section{References}

[1] G. G. Walter, "A sampling theorem for wavelet subspaces," IEEE Transactions on Information Theory, vol. 38, no. 2, part 2, pp. 881-884, 1992.

[2] W. Chen, S. Itoh, and J. Shiki, "A sampling theorem for shift-invariant spaces," IEEE Transactions on Signal Processing, vol. 46, no. 3, pp. 2802-2810, 1998.

[3] X.-G. Xia and Z. Zhang, "On sampling theorem, wavelets, and wavelet transforms," IEEE Transactions on Signal Processing, vol. 41, no. 12, pp. 3524-3535, 1993.

[4] A. J. E. M. Janssen, "Zak transform and sampling theorems for wavelet subspaces," IEEE Transactions on Signal Processing, vol. 41, no. 12, pp. 3360-3364, 1993.

[5] Y. Liu, "Irregular sampling for spline wavelet subspaces," IEEE Transactions on Information Theory, vol. 42, no. 2, pp. 623-627, 1996. 
[6] J. S. Geronimo, D. P. Hardin, and P. R. Massopust, "Fractal functions and wavelet expansions based on several scaling functions," Journal of Approximation Theory, vol. 78, no. 3, pp. 373-401, 1994.

[7] I. W. Selesnick, "Interpolating multiwavelet bases and the sampling theorem," IEEE Transactions on Signal Processing, vol. 47, no. 6, pp. 1615-1621, 1999.

[8] W. Sun and X. Zhou, "Sampling theorem for multiwavelet subspaces," Chinese Science Bulletin, vol. 44, no. 14, pp. 1283-1286, 1999.

[9] D.-X. Zhou, "Interpolatory orthogonal multiwavelets and refinable functions," IEEE Transactions on Signal Processing, vol. 50, no. 3, pp. 520-527, 2002.

[10] C. Zhao and P. Zhao, "Sampling theorem and irregular sampling theorem for multiwavelet subspaces," IEEE Transactions on Signal Processing, vol. 53, no. 2, part 1, pp. 705-713, 2005.

[11] A. Aldroubi, Q. Sun, and W.-S. Tang, "Non-uniform sampling in multiply generated shift-invariant subspaces of $L^{p}\left(R^{d}\right), "$ in Wavelet Analysis and Applications, vol. 25 of AMS/IP Studies in Advanced Mathematics, pp. 1-8, American Mathematical Society, Providence, RI, USA, 2002.

[12] O. Christensen, An Introduction to Frames and Riesz Bases, Applied and Numerical Harmonic Analysis, Birkhäuser, Boston, Mass, USA, 2003.

[13] W. Rudin, Real and Complex Analysis, McGraw-Hill, New York, NY, USA, 3rd edition, 1987.

[14] W. Sun and X. Zhou, "Sampling theorem for wavelet subspaces: error estimate and irregular sampling," IEEE Transactions on Signal Processing, vol. 48, no. 1, pp. 223-226, 2000.

[15] X. Zhou and W. Sun, "On the sampling theorem for wavelet subspaces," The Journal of Fourier Analysis and Applications, vol. 5, no. 4, pp. 347-354, 1999.

[16] C. Blanco, C. Cabrelli, and S. Heineken, "Functions in sampling spaces," Sampling Theory in Signal and Image Processing, vol. 5, no. 3, pp. 275-295, 2006. 


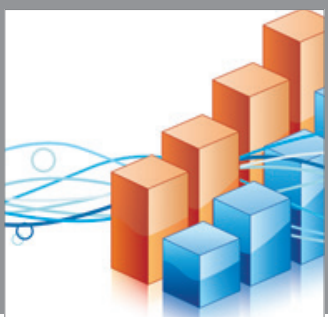

Advances in

Operations Research

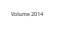

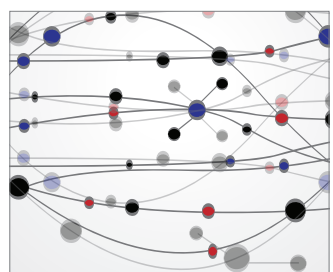

\section{The Scientific} World Journal
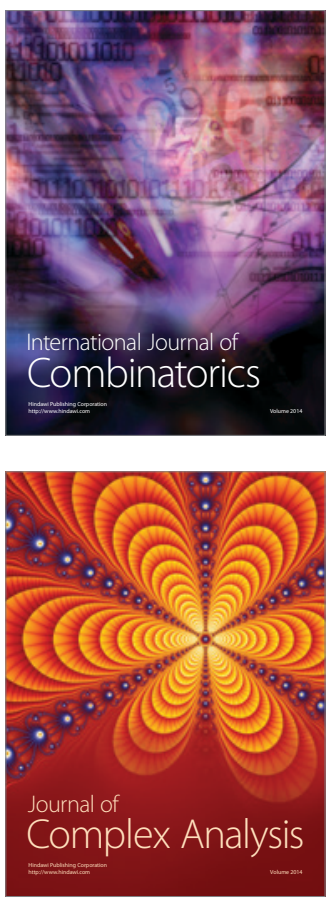

International Journal of

Mathematics and

Mathematical

Sciences
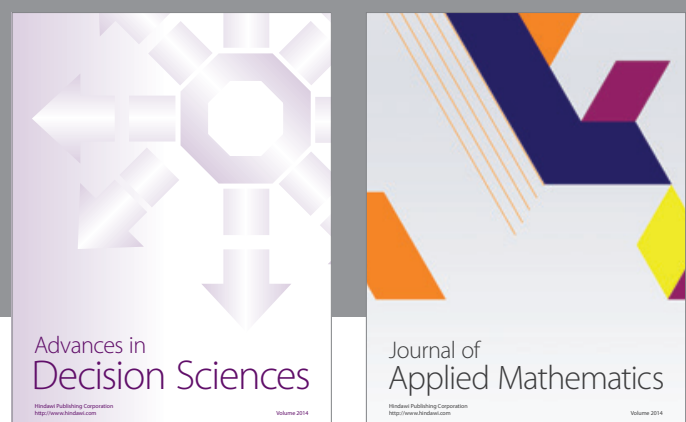

Journal of

Applied Mathematics
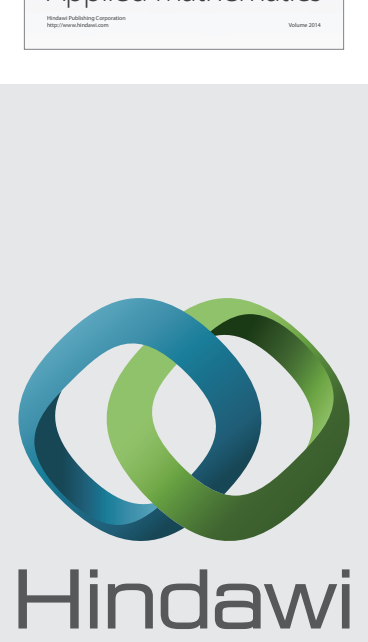

Submit your manuscripts at http://www.hindawi.com
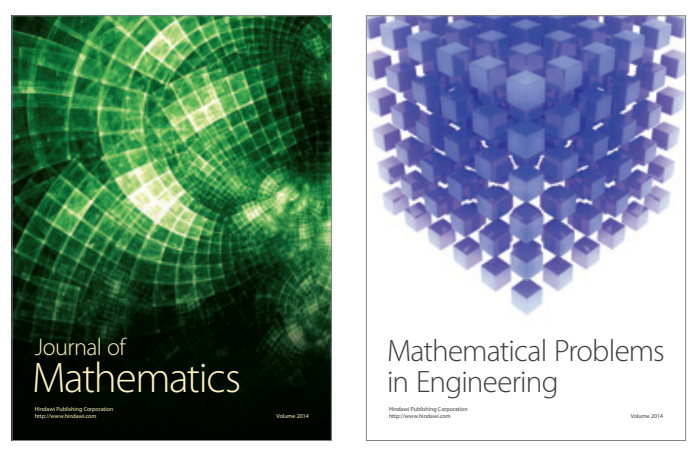

Mathematical Problems in Engineering
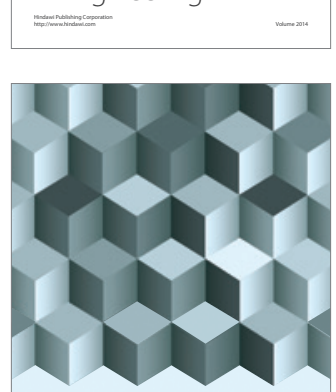

Journal of

Function Spaces
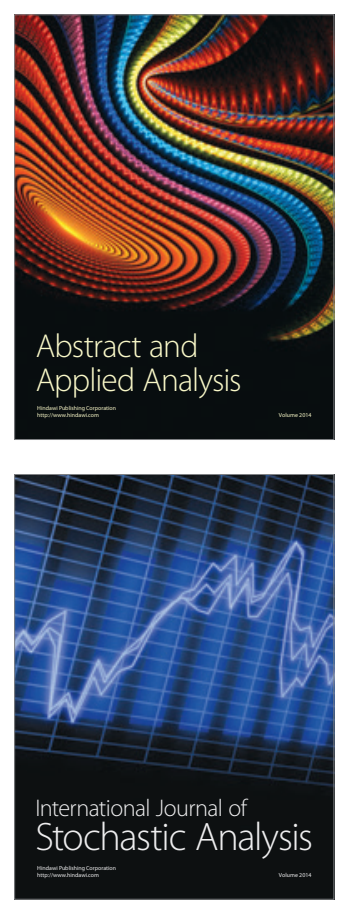

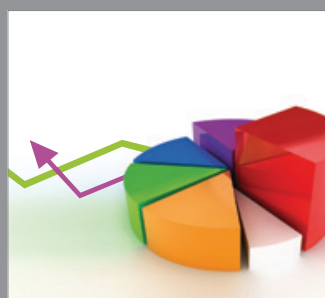

ournal of

Probability and Statistics

Promensencen
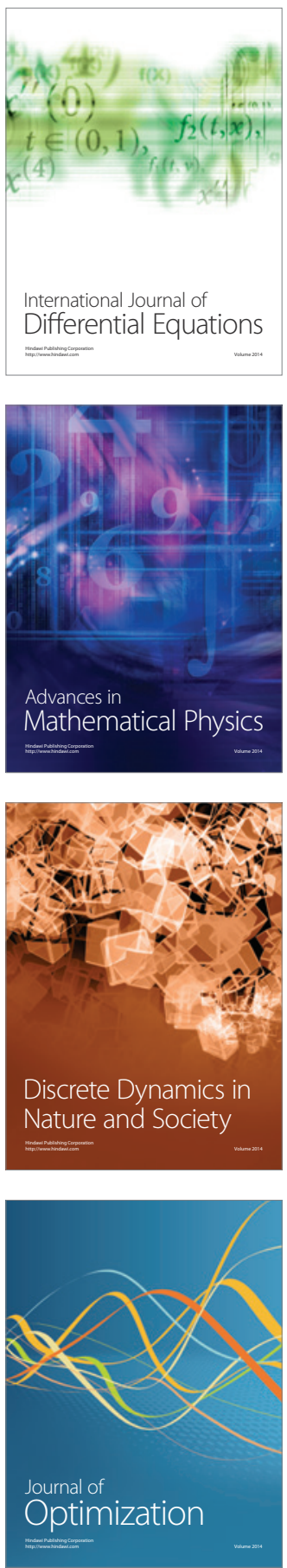